\title{
Establishment and evaluation of a nomogram model for predicting hematoma expansion in hypertensive intracerebral hemorrhage based on clinical factors and plain CT scan signs
}

\author{
Fei Yu ${ }^{1,2 \#}$, Yanli Yang ${ }^{1 \#}$, Yulun He ${ }^{1}$, Junwei Liu ${ }^{1}$, Haijun Liu $^{3}$, Heng Liu ${ }^{1}$ \\ ${ }^{1}$ Department of Radiology, Affiliated Hospital of Zunyi Medical University, Zunyi, China; ${ }^{2}$ Department of Medical Imaging, Chongqing University \\ Central Hospital, The Fourth People's Hospital of Chongqing, Chongqing, China; ${ }^{3}$ Department of Neurology, Affiliated Hospital of Zunyi Medical \\ University, Zunyi, China \\ Contributions: (I) Conception and design: F Yu, Heng Liu; (II) Administrative support: Heng Liu; (III) Provision of study materials or patients: F Yu, \\ Y Yang, Y He; (IV) Collection and assembly of data: F Yu, Y Yang, Y He; (V) Data analysis and interpretation: F Yu, J Liu, Haijun Liu, Heng Liu; (VI) \\ Manuscript writing: All authors; (VII) Final approval of manuscript: All authors. \\ \#These authors contributed equally to this work as co-first authors. \\ Correspondence to: Heng Liu; Junwei Liu. Department of Radiology, Affiliated Hospital of Zunyi Medical University, Zunyi, China. \\ Email: zmcliuh@163.com; zmcljw@163.com; Haijun Liu. Department of Neurology, Affiliated Hospital of Zunyi Medical University, Zunyi, China. \\ Email: 63258787@qq.com.
}

Background: Hematoma expansion (HE) is an important risk factor for poor prognosis in patients with hypertensive intracerebral hemorrhage. This study aimed to establish a nomogram model for predicting HE, and evaluate the model.

Methods: The clinical data and plain computed tomography (CT) scan signs of 341 patients with hypertensive intracerebral hemorrhage were retrospectively analyzed. According to the development of $\mathrm{HE}$, the patients were divided into an HE group (100 cases) and a non-HE group (241 cases). The clinical data and CT scan signs of the patients in these two groups were compared. Variables that had statistically significant differences were included in the multivariate logistic regression analysis to screen for independent predictors of $\mathrm{HE}$ and establish a nomogram model. The discrimination, calibration, and clinical practicability of this model were evaluated using the receiver operating characteristic (ROC) curve, calibration curve, and a decision curve analysis (DCA), respectively. Finally, the internal validation of this model was performed using the bootstrap method.

Results: The time interval from disease onset to the first CT [odds ratio $(\mathrm{OR})=0.807,95 \%$ confidence interval (CI): 0.665-0.979], volume of the hematoma at the first CT (OR =1.017, 95\% CI: 1.001-1.033), irregular shape of the hematoma (OR =2.458, 95\% CI: 1.355-4.456), swirl sign (OR =2.308, 95\% CI: 1.239 4.298), and blend sign ( $\mathrm{OR}=2.509,95 \% \mathrm{CI}$ : 1.304-4.830) were independent predictors of $\mathrm{HE}$ (all $\mathrm{P}<0.05$ ). These factors were used to establish a nomogram model. The area under the ROC curve of the model was 0.762 (95\% CI: 0.703-0.821). The results of the Hosmer-Lemeshow test and calibration curves showed that the predictive probabilities of the model fit the actual probabilities well. The DCA results showed that the domain probability range of the model was wide. The internal validation results showed that the $\mathrm{C}$-index was 0.751, and the model's discrimination was good.

Conclusions: The nomogram model established in this study had good discrimination, calibration, and clinical practicability. The model could serve as an intuitive and reliable guiding tool for the clinical identification of $\mathrm{HE}$ risk of hypertensive intracerebral hemorrhage.

Keywords: Hypertensive intracerebral hemorrhage; hematoma expansion (HE); prediction model; nomogram 
Submitted Sep 30, 2021. Accepted for publication Dec 16, 2021.

doi: 10.21037/apm-21-3569

View this article at: https://dx.doi.org/10.21037/apm-21-3569

\section{Introduction}

Intracerebral hemorrhage is the second most common form of cerebral stroke, and accounts for $10-20 \%$ of all cerebral strokes. The mortality rate of patients within 1 month of cerebral hemorrhage is as high as $40 \%$ (1). Hypertensive intracerebral hemorrhages account for $50-70 \%$ of all spontaneous intracerebral hemorrhages, and the incidence of hypertensive intracerebral hemorrhages continues to increase as the population ages (2). The main causes of hypertensive intracerebral hemorrhage are cerebrovascular atherosclerosis, hyaline degeneration, micro aneurysm formation and focal dilatation caused by long-term hypertension (1). Hematoma expansion (HE) is an important risk factor for the poor prognosis of patients with intracerebral hemorrhage, and has an incidence of approximately $13-38 \%$ (3). In the mechanism of $\mathrm{HE}$, persistent bleeding at the primary bleeding site or peripheral vascular injury bleeding is the main cause of HE (4). The development of HE is closely associated with neurological deterioration. It has been reported that a $1 \mathrm{~mL}$ increase in hematoma volume is associated with a $5 \%$ [95\% confidence interval (CI): 2-9\%] higher risk of death or dependency (5). Thus, the prevention of HE is an important treatment strategy for patients with intracerebral hemorrhage.

At present, the variables that have been proposed as predictors of $\mathrm{HE}$ include clinical factors, such as systolic blood pressure, the Glasgow Coma Scale (GCS) score, and the use of anti-platelet and anti-coagulant drugs (3), laboratory indicators, including coagulation function status, platelet level, blood glucose, and low-density lipoprotein cholesterol (LDL-C) level (6), and imaging signs, such as an irregular hematoma shape, heterogeneous hematoma density, black hole sign, swirl sign, blend sign, island sign, satellite sign, fluid level sign, and computed tomography (CT)-angiography (CTA) spot sign (7). However, regardless of whether a clinical factor, a laboratory indicator, or an imaging sign is used, it is still challenging to predict the occurrence of HE using a single factor, and doing so reduces the sensitivity and specificity of predicting HE.

Nomogram is a quantitative analysis diagram that uses a cluster of disjoint line segments to represent the functional relationship between multiple variables in cartesian coordinate system. It is the visualization of the results of logistic regression and Cox regression equation. It can directly use the graph to calculate the incidence of an event, which has been widely applied to oncology research (8). At present, there are only a few studies on the prediction of HE in hypertensive intracerebral hemorrhage by nomogram model, for example, Zhang et al. (9) incorporated the time of initial CT, NIHSS score, CTA spot sign, hypodensities, blend sign and international normalized ratio (INR) to build a nomogram model, in which CTA spot sign plays an important role. However, when some patients cannot perform CTA due to renal insufficiency or contrast medium allergy, the model has limited application.

Thus, this study aimed to comprehensively use clinical factors, laboratory indicators, and plain CT scan imaging signs to establish a clinical prediction model for the prediction of HE. Each index can be obtained after completing CT plain scan, which is more convenient and quicker. This model was visualized using a nomogram and was evaluated from multiple angles.

We present the following article in accordance with the TRIPOD reporting checklist (available at https://dx.doi. org/10.21037/apm-21-3569).

\section{Methods}

\section{Patient selection}

The data of patients with hypertensive intracerebral hemorrhage treated at the Chongqing University Central Hospital, China between January 2017 and July 2021 were retrospectively and continuously collected using the Picture Archiving and Communication Systems (PACS).

\section{Inclusion criteria}

Patients were eligible for inclusion in the study if they met the following inclusion criteria:

(I) Had an accurate history of hypertension (hypertension was defined as systolic pressure $\geq 140 \mathrm{mmHg}$ and/or diastolic pressure $\geq 90 \mathrm{mmHg}$ ) (10);

(II) Had received their first CT scan within $6 \mathrm{~h}$ of disease onset; 


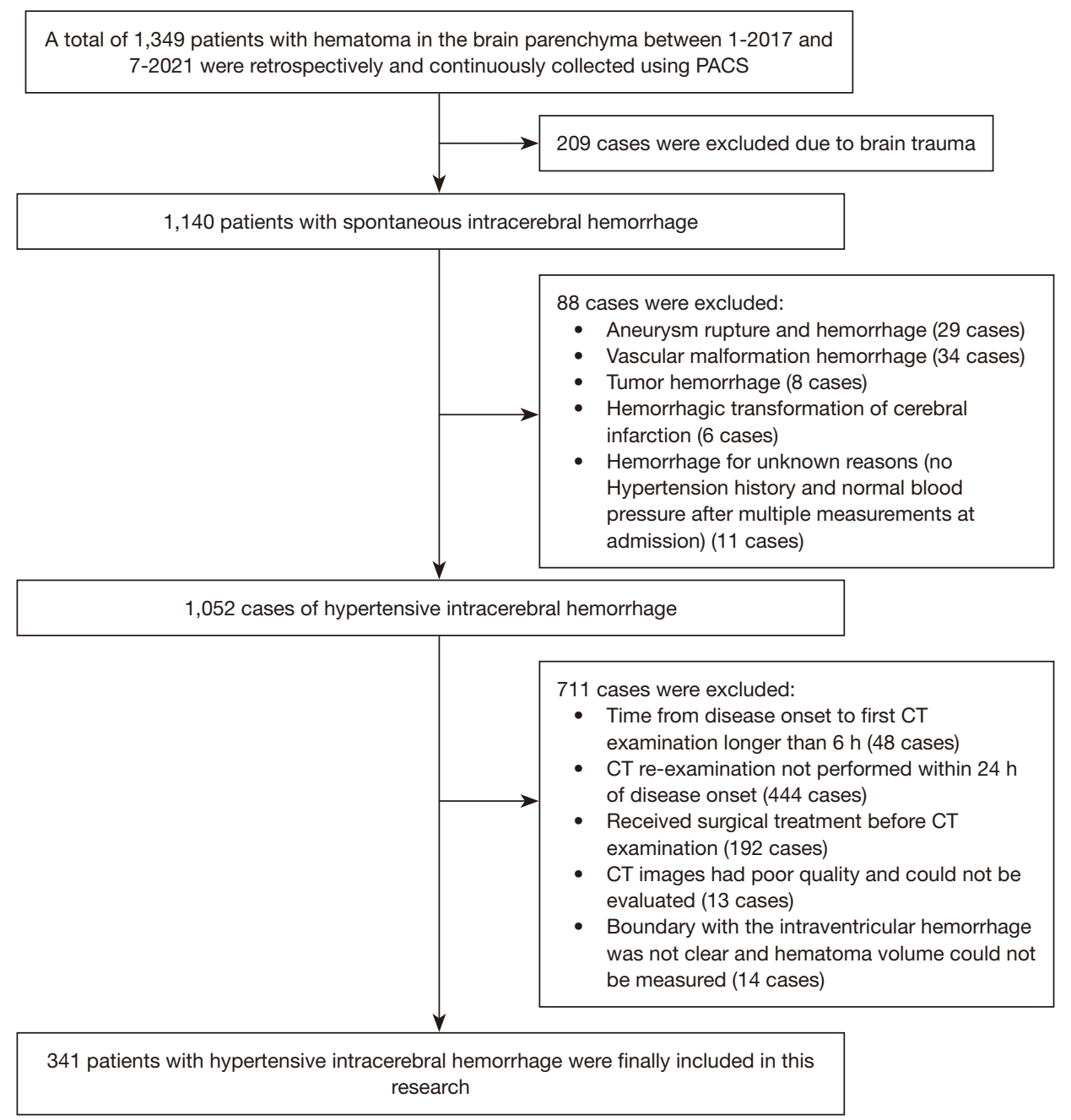

Figure 1 The flow chart for patient screening. PACS, Picture Archiving and Communication Systems; CT, computed tomography.

(III) Had completed a CT re-examination within $24 \mathrm{~h}$ of disease onset; and

(IV) Had a hemorrhage that was located in the brain parenchyma, including the basal ganglia, thalamus, brain lobes, brainstem, and cerebellum.

\section{Exclusion criteria}

Patients were excluded from the study if they met any of the following exclusion criteria:

(I) Had secondary intracerebral hemorrhage caused by brain trauma, cerebrovascular malformations, aneurysms, and tumors;

(II) $\mathrm{Had}$ ischemic cerebral infarction hemorrhagic transformation;
(III) Had a primary intraventricular hemorrhage;

(IV) Had undergone surgical treatment (e.g., hematoma removal or puncture drainage) before the CT examination; and/or;

(V) Had images of poor quality that could not be accurately evaluated.

The patient screening process is shown in Figure 1. All procedures performed in this study involving human participants were in accordance with the Declaration of Helsinki (as revised in 2013). Our research was approved by the Ethics Committee of The Fourth People's Hospital of Chongqing (No. 2021-62). This study was a retrospective study and was exempted from signing informed consent. 


\section{Instrument and image collection}

Image collection was performed using Lightspeed VCT 64-slice spiral CT (GE) and UCT760 64-slice spiral CT (United Imaging) for the cranial plain CT scan. The scan range was from the base of the skull to the top of the skull, the slice thickness was $5 \mathrm{~mm}$, the slice interval was $0 \mathrm{~mm}$, the tube voltage was $120 \mathrm{KV}$, and the tube current was 200-238 mAs.

\section{Hematoma volume calculation and patient grouping}

The ABC/2 Coniglobus formula was used to calculate hematoma volume $(11,12)$, where $\mathrm{A}$ is the longest diameter of the largest layer of the hematoma on the axial CT images, $\mathrm{B}$ is the longest diameter perpendicular to $\mathrm{A}$ in that layer, and $\mathrm{C}$ is the depth of the hematoma perpendicular to that layer. The hematoma volume at the first CT scan (V1) and the hematoma volume at the last CT examination within 24 h of disease onset (V2) were calculated separately.

Definition of $\mathrm{HE}$ and patient grouping: According to the standard definition for HE proposed in literature $(13,14)$, $\mathrm{HE}$ was defined as a V2 that is larger than V1 by more than $33 \%$ or an absolute increase greater than $6 \mathrm{~mL}$. The patients were divided into the $\mathrm{HE}$ and non-HE groups.

\section{Observation indicators}

Clinical data included the patients' age and sex, GCS score at admission, systolic pressure, diastolic pressure, diabetes mellitus history, smoking history, past cerebral stroke history, and time from disease onset to the first CT. Laboratory indicators included the platelet count within $24 \mathrm{~h}$ of admission, activated partial thromboplastin time, international normalized ratio, and fibrinogen, urea, creatinine, uric acid, C-reactive protein, highdensity lipoprotein cholesterol (HDL-C), LDL-C, and apolipoprotein A1 (ApoA1) levels. Plain CT scan signs included the hematoma volume at the first CT, hematoma location, irregular hematoma shape, heterogeneous hematoma density, black hole sign, swirl sign, blend sign, island sign, satellite sign, fluid level sign, degree of cerebral midline displacement, combined ventricular system hemorrhage, and subarachnoid hemorrhage. The identification and interpretation of CT plain scan signs were based on the definitions and requirements of the Standards for Detecting, Interpreting, and Reporting Noncontrast Computed Tomographic Markers of Intracerebral
Hemorrhage Expansion (7). Hematoma volume measurement and observation and the identification of plain CT scan signs were independently performed by 2 neuroimaging physicians with 5 years of experience under double-blinded conditions. When the results were not consistent, the 2 physicians discussed them until a consensus was reached.

When the missing values for an observation indicator data were $\geq 20 \%$, the observation indicator was excluded. When the missing values were $<20 \%$, they were filled in using the average value for the sequence.

\section{Statistical analysis}

Statistical analyses were performed using SPSS (version 26.0). The receiver operating characteristic (ROC) curve, nomogram, calibration chart, decision curve analysis (DCA), and internal validation were completed using $\mathrm{R}$ software (version 4.0.0). The normality of all the measurement data was tested using the Kolmogorov-Smirnov method. Continuous variables that conformed to a normal distribution are expressed as mean \pm standard deviation $(\bar{x} \pm s)$, and were examined using the independent samples $t$-test. Continuous variables that did not conform to a normal distribution are expressed as median (interquartile range), and were examined using the Mann-Whitney $\mathrm{U}$ test. Categorical variables are expressed as frequency (percentage), and were examined using the chi-square test or Fisher's exact test. The kappa test was used to evaluate the consistency of $\mathrm{HE}$ as determined by the 2 physicians using the measurement of hematoma volume.

The clinical data and CT plain scan signs of patients with HE and non-HE groups were compared, and the statistically significant variables were included in multivariate logistic regression. A backward stepwise regression was performed based on the principle of the least Akaike information criterion (AIC) to screen independent predictors associated with HE. Next, the rms package in $\mathrm{R}$ software was used to establish a nomogram model for predicting HE.

The ROC curve and Hosmer-Lemeshow test (for which a $\mathrm{P}$ value $>0.05$ indicated a good fit) were used to evaluate the discrimination and calibration abilities of the model. The model's goodness of fit was depicted using the calibration curve. The model's net benefit was evaluated using DCA to guide clinical decision making. The area under the ROC curve (AUC), diagnostic accuracy, sensitivity, specificity, positive predictive value (PPV), and negative predictive value (NPV) of the model were calculated, and a nomogram 
was plotted to visualize the model's results. Internal validation of the model was performed using 1,000-times bootstrapping, and the consistency index (C-index) was calculated. The test was bilateral at a level of $\alpha=0.05$.

\section{Results}

\section{Comparison of the clinical data and plain CT scan signs of the patients in the two groups}

A total of 341 patients were included in this research. There were 100 patients (29.3\%) in the HE group and 241 patients (70.7\%) in the non-HE group. The 2 physicians' findings of HE were mostly consistent, and the kappa value was 0.901 . The comparison of the two groups showed statistically significant differences in the time from disease onset to the first CT scan, hematoma volume at the first CT, GCS score at admission, irregular hematoma shape, heterogeneous hematoma density, swirl sign, blend sign, black hole sign, island sign, satellite sign, fluid level sign, degree of cerebral midline displacement, and subarachnoid hemorrhage (all $\mathrm{P}<0.05$; see Table 1). The percentages of missing values for C-reactive protein, HDL-C, LDL-C, and ApoA1 were $36.9 \%, 31.4 \%, 31.4 \%$, and $31.4 \%$, respectively. There were many missing values (all $>20 \%$ ); thus, these observation indicators were excluded.

\section{Multivariate logistic regression analysis of $\mathrm{HE}$}

Variables that differed significantly between the two groups were included in the multivariate logistic regression analysis. Screening using the backward stepwise regression based on the principle of the least AIC (AIC =350.91) showed that the time interval from disease onset to the first CT scan, hematoma volume at the first CT, irregular hematoma shape, swirl sign, and blend sign were independent predictors of $\mathrm{HE}$ (all $\mathrm{P}<0.05$; see Table 2).

\section{Establishment and evaluation of the nomogram model}

The rms package in $\mathrm{R}$ software was used to establish the nomogram model for the prediction of HE (see Figure 2). The score for each independent predictor corresponded to values on the upper side of the scoring scale. The total score for each patient was the sum of the score value for each independent predictor. The total score corresponded to the value on the risk axis, which indicated the risk of developing HE. The risk of developing HE was higher when the total score was higher.

The AUC of this nomogram model was $0.762(95 \%$ CI: $0.703-0.821)$. The prediction effect of this model was good (see Figure 3). The accuracy of this model for predicting HE was $79.5 \%$, the sensitivity was $49.0 \%$, the specificity was $92.1 \%$, the PPV was $72.1 \%$, and the NLR was $81.3 \%$. The Hosmer-Lemeshow test $\left(\chi^{2}=14.61\right.$; $\mathrm{P}=0.067$ ) and calibration curve showed that the probability predicted by this model had a high degree of fit to the actual probability (see Figure 4). A DCA was conducted to evaluate the clinical practicability of this nomogram model. This nomogram model had a large domain probability range (Figure 5), indicating that its clinical practicability was strong. The internal validation results showed that the C-index was 0.751 , and the discrimination level of this model was excellent. Collected 2 patients with hypertensive intracerebral hemorrhage in August 2021, and applied this nomogram model to them to predict the risk of hematoma expansion (Figures 6,7).

\section{Discussion}

This research examined clinical factors, laboratory indicators, and CT plain scan signs, and found that the time from the disease onset to the first CT scan, hematoma volume at the first CT scan, irregular hematoma shape, the swirl sign, and the blend sign were independent predictors of the development of early $\mathrm{HE}$ in patients with hypertensive intracerebral hemorrhage. Additionally, a clinical prediction model was established and presented in the form of a nomogram. A multiangle valuation of this model showed that its discrimination, calibration, and clinical practicability were all good. The developed model could be used as an intuitive and reliable tool for the early clinical identification of patients at risk of developing $\mathrm{HE}$.

Hypertensive intracerebral hemorrhage is a spontaneous non-traumatic intracerebral hemorrhage. It is the common cerebrovascular disease among middle-aged and elderly people (15). It has very high morbidity and mortality, and its incidence is increasing yearly with the aging of the population $(2,16)$. Some studies have shown that hypertension is more likely to be associated with a large intracranial hematoma volume and the development of HE. In addition, controlling the systolic blood pressure at $50 \mathrm{mmHg}$ could reduce the risk of HE $(3,17)$. Lord et al. (18) showed that the development of neurological deterioration in the hyperacute (within $1 \mathrm{~h}$ ) and acute (within $24 \mathrm{~h}$ ) periods after intracerebral hemorrhage was associated with 
Table 1 Comparison of the clinical data and plain CT scan signs of patients with hypertensive intracerebral hemorrhage in the HE group and the non-HE group

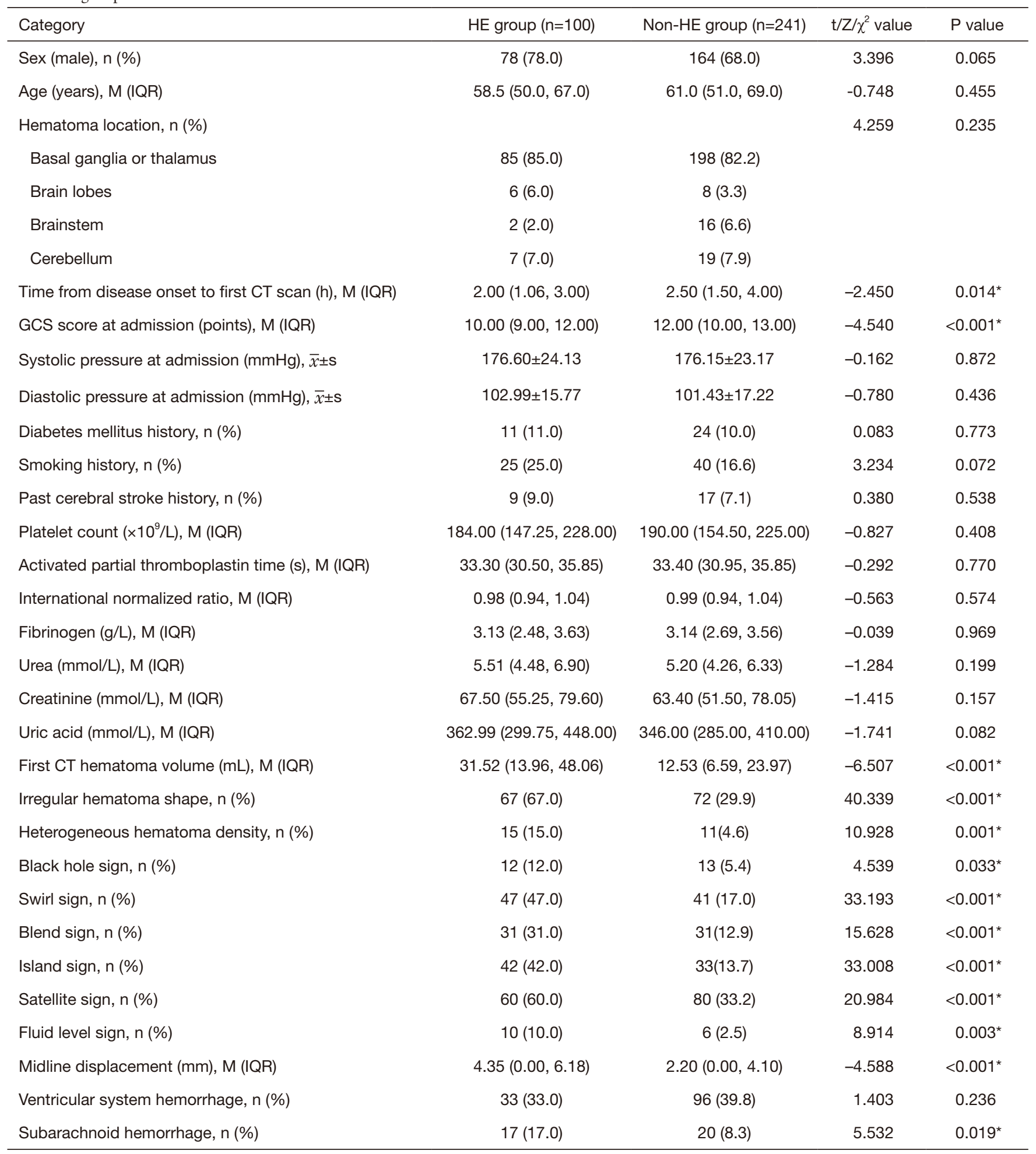

*, $\mathrm{P}<0.05$, indicating that the difference was statistically significant. HE, hematoma expansion; CT, computed tomography; GCS, Glasgow Coma Scale; M, median; IQR, interquartile range; $\bar{x}$, mean; s, standard deviation. 
Table 2 Multivariate logistic regression analysis of factors related to HE

\begin{tabular}{|c|c|c|c|c|c|}
\hline Factor & $B$ value & SE & Wald $\chi^{2}$ value & OR value $(95 \% \mathrm{Cl})$ & $P$ value \\
\hline Hematoma volume on first CT scan (mL) & 0.017 & 0.008 & 4.521 & $1.017(1.001,1.033)$ & $0.033^{*}$ \\
\hline Irregular hematoma shape & 0.899 & 0.304 & 8.772 & $2.458(1.355,4.456)$ & $0.003^{*}$ \\
\hline Swirl sign & 0.836 & 0.317 & 6.947 & $2.308(1.239,4.298)$ & $0.008^{*}$ \\
\hline
\end{tabular}

*, $\mathrm{P}<0.05$, indicating that the difference was statistically significant. HE, hematoma expansion; CT, computed tomography; B, regression coefficient; SE, standard error; OR, odds ratio; Cl, confidence interval.

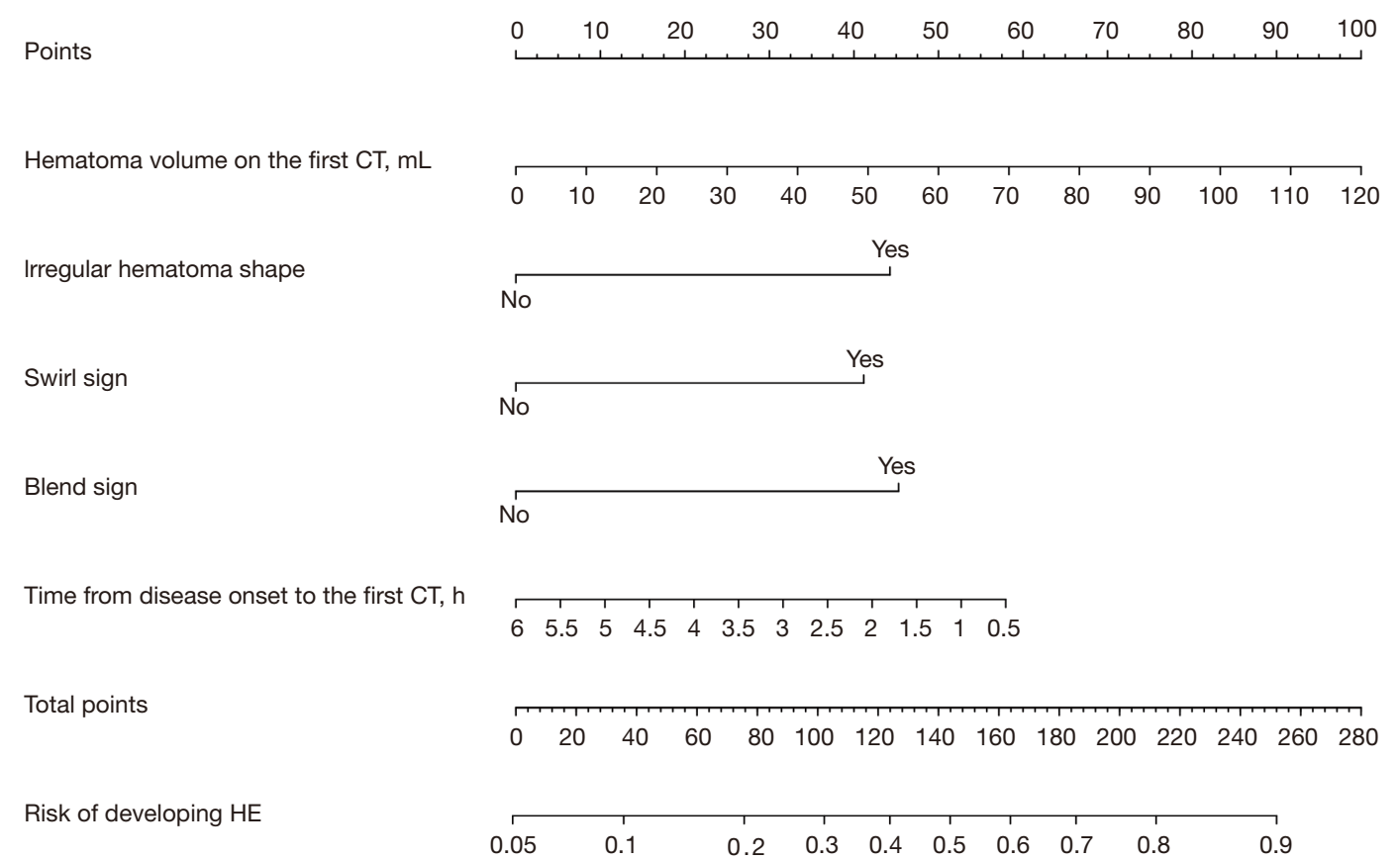

Figure 2 The nomogram for predicting HE in hypertensive intracerebral hemorrhage patients. HE, hematoma expansion. CT, computed tomography; mL, milliliter; h, hour.

HE (ORs $=3.60$ and 7.59, respectively, and 95\% CIs: $1.76-$ 7.6 and 3.91-14.74, respectively). Therefore, preventing $\mathrm{HE}$ is one of the important strategies for the treatment of patients with hypertensive intracerebral hemorrhage. At present, the prevention of HE mainly focuses on blood pressure control, hemostatic treatment and blood glucose management (3), among which the control of blood pressure plays an important role. Li et al. (19) found that ultra-early intensive blood pressure reduction treatment of intracerebral hemorrhage was related to reducing the risk of $\mathrm{HE}(\mathrm{OR}=0.56,95 \% \mathrm{CI}: 0.34-0.92)$. However, high-intensity antihypertensive therapy can lead to ischemia of brain tissue around and far away from the hematoma (20), and reduce the benefits of intensive blood pressure reduction treatment to patients. Therefore, targeted selection of patients with high risk of HE is key for intensive antihypertensive treatment. Thus, predicting the risk of developing HE has important clinical significance for the treatment of hypertensive intracerebral hemorrhage.

Previous studies indicated that HE is associated with many risk factors. The CTA spot sign is an independent risk factor for HE. Demchuk et al. (21) showed that the CTA spot sign had $51 \%$ sensitivity and $85 \%$ specificity in the prediction of $\mathrm{HE}$, and included it in a 9-point scoring 


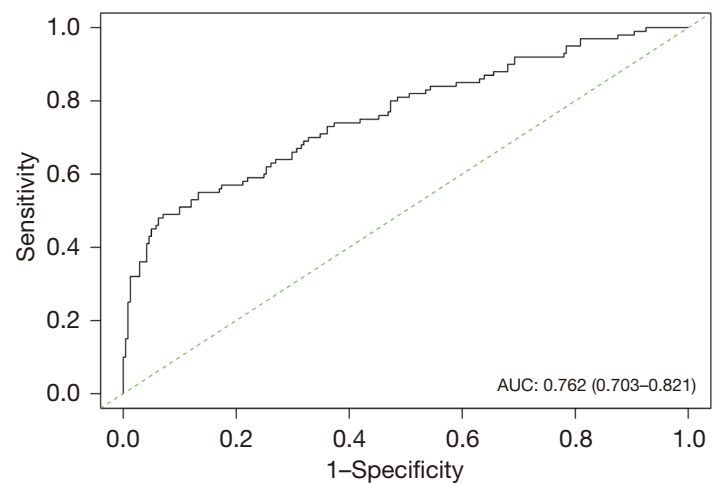

Figure 3 The ROC curve of the nomogram model for predicting $\mathrm{HE}$ in hypertensive intracerebral hemorrhage patients. ROC, receiver operating characteristic; $\mathrm{HE}$, hematoma expansion; AUC, area under curve.

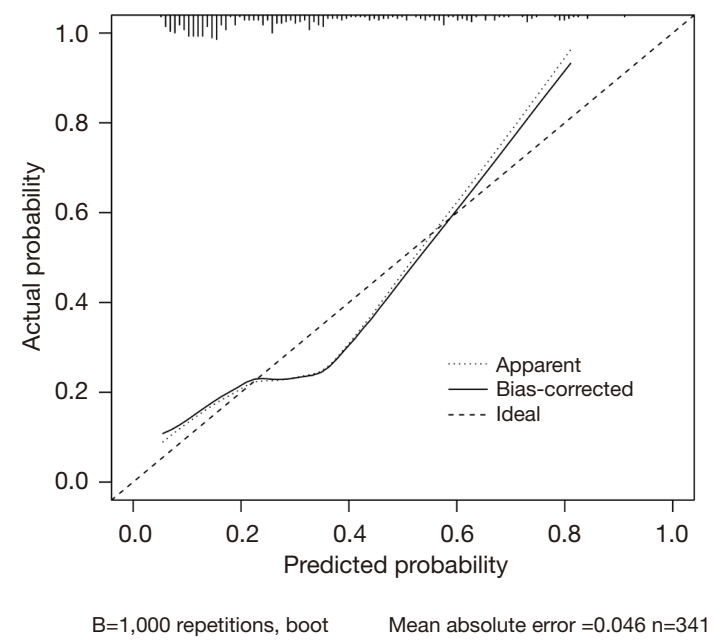

Figure 4 The calibration curve of the nomogram model for predicting $\mathrm{HE}$ in hypertensive intracerebral hemorrhage patients. HE, hematoma expansion.

system (22). However, because in clinical practice, CTA images cannot always be acquired in a timely manner for reasons such as a contrast agent allergy, renal insufficiency, or patient non-cooperation during examination, its inclusion in a scoring system for the prediction of $\mathrm{HE}$ would reduce the timeliness and effectiveness of the system. All 5 of the predictors included in this study can be acquired immediately after the completion of the first CT scan and are faster and more convenient to acquire than CTA signs.

In this research, the time from disease onset to the first CT scan referred to the interval between the onset

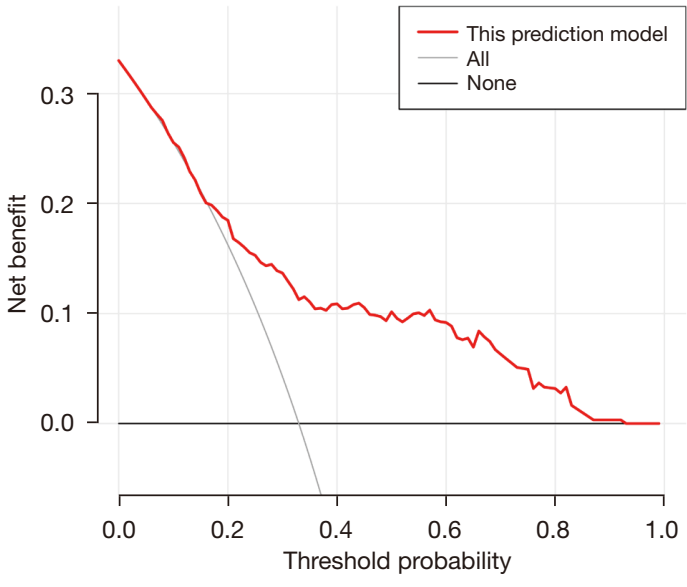

Figure 5 The DCA curve of the nomogram model for predicting $\mathrm{HE}$ in hypertensive intracerebral hemorrhage patients. DCA, decision curve analysis; HE, hematoma expansion.

of clinical symptoms and the first CT examination. Many previous studies have confirmed that the time from disease onset to the first CT scan is an independent predictor of $\mathrm{HE}(9,23,24)$. HE after an intracerebral hemorrhage usually occurs 3-6 h after the hemorrhage. After $24 \mathrm{~h}$, the hematoma volume is usually stable (25). The possibility of detecting an unstable hematoma on CT examination is higher in the early stage after disease onset; thus, the possibility of detecting HE in the subsequent CT reexamination is also higher. The nomogram model in this study showed that when the time from disease onset to the first CT scan was shorter, the model score was higher, and the probability of HE development was higher. The hematoma volume at the first CT scan was associated with the development of HE. Many studies indicated that the hematoma volume at the first CT scan was an independent predictor of HE $(14,24,26)$ and was closely associated with the patient's prognosis and neurological deterioration (18). Under the HE clinical prediction scoring system, both the time from disease onset to the first CT scan and the hematoma volume at the first CT scan were included in the 9-point and 24-point scoring systems $(22,27)$.

The plain CT scan signs included in this model, including the irregular hematoma shape, swirl sign, and blend sign, were all significantly associated with HE. In terms of the mechanism of HE development, persistent hemorrhage at the original bleeding site or secondary hemorrhage from peripheral vascular injury were major reasons for HE (28). Barras et al. (29) showed that irregular hematoma shape was associated with secondary 

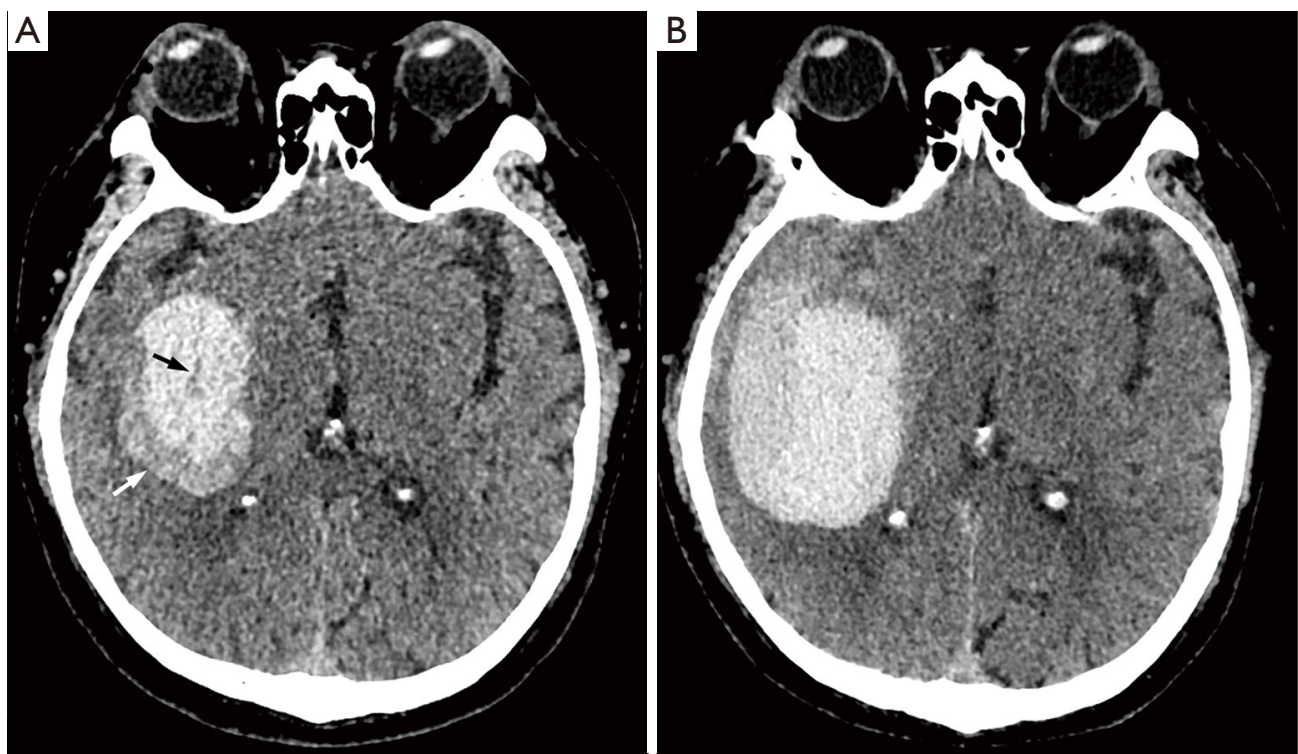

Figure 6 Brain CT plain scan of patient with hypertensive intracerebral hemorrhage, male, 65 years old. (A) He was admitted to the hospital 1 hour after onset. The volume of hematoma was about $61 \mathrm{~mL}$, regular hematoma shape, and swirl sign (black arrow) and blend sign (white arrow) were visible. The scores of 5 items in the nomogram were 50, 0, 41, 45, and 52.5 respectively. Total score is 188.5 and the risk of developing hematoma expansion is $71 \%$. (B) CT re-examination 10 hours after onset, the hematoma volume increased. CT, computed tomography.
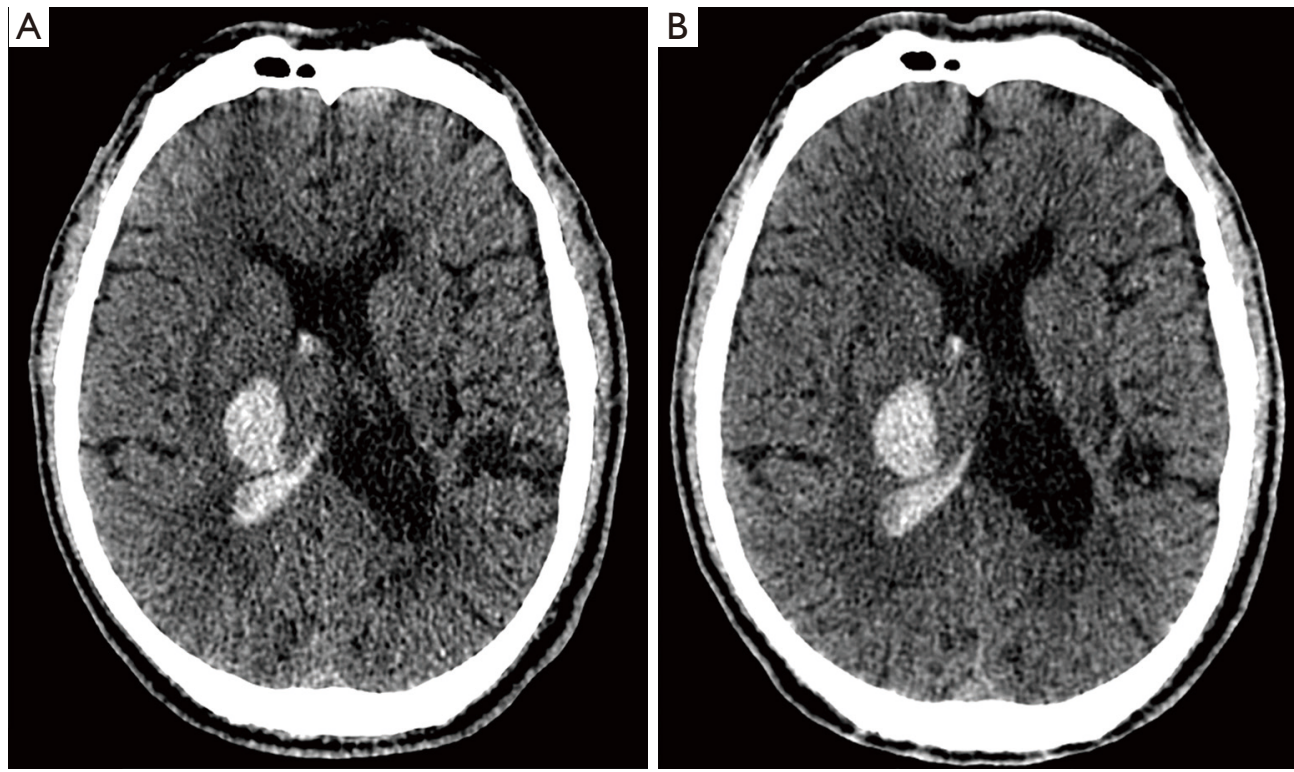

Figure 7 Brain CT plain scan of patient with hypertensive intracerebral hemorrhage, male, 70 years old. (A) He was admitted to the hospital 5 hours after onset. The hematoma volume was about $6 \mathrm{~mL}$, with regular hematoma shape and no swirl sign and blend sign. The scores of 5 items in the nomogram were 5, 0, 0, 0 and 11 respectively. Total score is 16 and the risk of developing hematoma expansion is $7 \%$. (B) CT re-examination 11.5 hours after onset showed that the hematoma volume did not increase. CT, computed tomography. 
hemorrhage at the hematoma margin. They formulated a 5 -point scoring system in which 1 point indicated that the hematoma had regular morphology and no lobes, 2 points indicated the presence of 1 lobe, and so on up to 5 points; 1-2 points indicated regular hematoma shapes, and 3-5 points indicated irregular hematoma shapes. The metaanalysis study of $\mathrm{Yu}$ et al. (30) showed that the prediction of $\mathrm{HE}$ using irregular hematoma shape had $67 \%$ sensitivity and $47 \%$ specificity. Thus, irregular hematoma shape can be used as a predictor of HE, but its specificity is low. The swirl sign is defined as the presence of 1 or more round, striped, or irregular low-density areas in high-density hematomas. It has been confirmed to be an independent predictor of HE in many studies $(24,31,32)$. A metaanalysis (33) showed that the swirl sign had $45 \%$ sensitivity and $79 \%$ specificity in the prediction of HE. Thus, its specificity is high in the prediction of HE. The blend sign is defined as the presence of relatively low- and high-density areas in hematomas. The boundary between the 2 types of areas is clear, and the density difference is $>18 \mathrm{Hu}$. It has a sensitivity of $13-42.86 \%$ and a specificity of $88.51-95.5 \%$, and many studies have confirmed that it is a risk factor for HE $(34,35)$.

Currently, there many HE prediction models and scoring systems available. However, there are fewer reports on the establishment of prediction models using the nomogram form. This research used a total of 5 indicators to establish a nomogram model. It included the clinical indicator of the time from disease onset to the first CT scan and the imaging indicators of hematoma volume at the first CT scan, irregular hematoma shape, the swirl sign, and the blend sign. All of these indicators can be acquired immediately after the completion of the first CT examination. Thus, this model is convenient, fast, and time sensitive. This research performed a multiangled evaluation of the discrimination, calibration, and clinical practicability of this model, and the results confirmed that the model is reliable and effective. In addition, this model is presented in the form of an intuitive nomogram that can be easily disseminated and applied in clinical practice and can help clinicians to quickly identify patients at risk of $\mathrm{HE}$ and take timely measures for monitoring and treatment. Thus, this model can improve patient prognosis and reduce the risk of neurological deterioration to some extent.

This research had a number of limitations. First, this was a single-center study, the number of positive cases was low, and there was no external validation. Thus, the number of cases should be increased, or further multicenter research should be conducted. Second, the clinical factors and laboratory indicators included in this research were limited; for example, laboratory indicators, such as blood lipid and C-reactive protein levels, were not included in this research because the percentages of missing values were large (missing rates $>20 \%$ ). Thus, some risk factors associated with $\mathrm{HE}$ might be not have been considered. Third, this was a retrospective study, and the follow-up of treatment protocols and prognosis was lacking. Thus, the results cannot be used to guide the formulation of clinical protocols or determine the prognosis of patients. Further prospective studies will be conducted to establish a model that can provide greater guidance for patient treatment.

\section{Acknowledgments}

Funding: None.

\section{Footnote}

Reporting Checklist: The authors have completed the TRIPOD reporting checklist. Available at https://dx.doi. org/10.21037/apm-21-3569

Data Sharing Statement: Available at https://dx.doi. org/10.21037/apm-21-3569

Conflicts of Interest: All authors have completed the ICMJE uniform disclosure form (available at https://dx.doi. org/10.21037/apm-21-3569). The authors have no conflicts of interest to declare.

Ethical Statement: The authors are accountable for all aspects of the work in ensuring that questions related to the accuracy or integrity of any part of the work are appropriately investigated and resolved. All procedures performed in this study involving human participants were in accordance with the Declaration of Helsinki (as revised in 2013). Our research was approved by the Ethics Committee of The Fourth People's Hospital of Chongqing (No. 2021-62). This study was a retrospective study and was exempted from signing informed consent.

Open Access Statement: This is an Open Access article distributed in accordance with the Creative Commons Attribution-NonCommercial-NoDerivs 4.0 International License (CC BY-NC-ND 4.0), which permits the noncommercial replication and distribution of the article with 
the strict proviso that no changes or edits are made and the original work is properly cited (including links to both the formal publication through the relevant DOI and the license). See: https://creativecommons.org/licenses/by-nc-nd/4.0/.

\section{References}

1. An SJ, Kim TJ, Yoon BW. Epidemiology, Risk Factors, and Clinical Features of Intracerebral Hemorrhage: An Update. J Stroke 2017;19:3-10.

2. Tang Y, Yin F, Fu D, et al. Efficacy and safety of minimal invasive surgery treatment in hypertensive intracerebral hemorrhage: a systematic review and meta-analysis. BMC Neurol 2018;18:136.

3. Li Z, You M, Long C, et al. Hematoma Expansion in Intracerebral Hemorrhage: An Update on Prediction and Treatment. Front Neurol 2020;11:702.

4. Guan J, Hawryluk GW. Targeting Secondary Hematoma Expansion in Spontaneous Intracerebral Hemorrhage State of the Art. Front Neurol 2016;7:187.

5. Delcourt C, Huang Y, Arima H, et al. Hematoma growth and outcomes in intracerebral hemorrhage: the INTERACT1 study. Neurology 2012;79:314-9.

6. Wang G, Zhang J. Hematoma Expansion: Clinical and Molecular Predictors and Corresponding Pharmacological Treatment. Curr Drug Targets 2017;18:1367-76.

7. Morotti A, Boulouis G, Dowlatshahi D, et al. Standards for Detecting, Interpreting, and Reporting Noncontrast Computed Tomographic Markers of Intracerebral Hemorrhage Expansion. Ann Neurol 2019;86:480-92.

8. Caulfield S, Menezes G, Marignol L, et al. Nomograms are key decision-making tools in prostate cancer radiation therapy. Urol Oncol 2018;36:283-92.

9. Zhang C, Ge H, Zhong J, et al. Development and validation of a nomogram for predicting hematoma expansion in intracerebral hemorrhage. J Clin Neurosci 2020;82:99-104.

10. Jones NR, McCormack T, Constanti M, et al. Diagnosis and management of hypertension in adults: NICE guideline update 2019. Br J Gen Pract 2020;70:90-1.

11. Morotti A, Goldstein JN. Diagnosis and Management of Acute Intracerebral Hemorrhage. Emerg Med Clin North Am 2016;34:883-99.

12. Hanley DF, Thompson RE, Rosenblum M, et al. Efficacy and safety of minimally invasive surgery with thrombolysis in intracerebral haemorrhage evacuation (MISTIE III): a randomised, controlled, open-label, blinded endpoint phase 3 trial. Lancet 2019;393:1021-32.
13. Song Z, Guo D, Tang Z, et al. Noncontrast Computed Tomography-Based Radiomics Analysis in Discriminating Early Hematoma Expansion after Spontaneous Intracerebral Hemorrhage. Korean J Radiol 2021;22:41524.

14. Hu S, Sheng W, Hu Y, et al. A nomogram to predict early hematoma expansion of hypertensive cerebral hemorrhage. Medicine (Baltimore) 2021;100:e24737.

15. Che XR, Wang YJ, Zheng HY. Prognostic value of intracranial pressure monitoring for the management of hypertensive intracerebral hemorrhage following minimally invasive surgery. World J Emerg Med 2020;11:169-73.

16. Feigin VL, Lawes CM, Bennett DA, et al. Worldwide stroke incidence and early case fatality reported in 56 population-based studies: a systematic review. Lancet Neurol 2009;8:355-69.

17. Ohwaki K, Yano E, Nagashima H, et al. Blood pressure management in acute intracerebral hemorrhage: relationship between elevated blood pressure and hematoma enlargement. Stroke 2004;35:1364-7.

18. Lord AS, Gilmore E, Choi HA, et al. Time course and predictors of neurological deterioration after intracerebral hemorrhage. Stroke 2015;46:647-52.

19. Li Q, Warren AD, Qureshi AI, et al. Ultra-Early Blood Pressure Reduction Attenuates Hematoma Growth and Improves Outcome in Intracerebral Hemorrhage. Ann Neurol 2020;88:388-95.

20. Kidwell CS, Rosand J, Norato G, et al. Ischemic lesions, blood pressure dysregulation, and poor outcomes in intracerebral hemorrhage. Neurology 2017;88:782-8.

21. Demchuk AM, Dowlatshahi D, Rodriguez-Luna D, et al. Prediction of haematoma growth and outcome in patients with intracerebral haemorrhage using the CT-angiography spot sign (PREDICT): a prospective observational study. Lancet Neurol 2012;11:307-14.

22. Huynh TJ, Aviv RI, Dowlatshahi D, et al. Validation of the 9-Point and 24-Point Hematoma Expansion Prediction Scores and Derivation of the PREDICT A/B Scores. Stroke 2015;46:3105-10.

23. Yao X, Xu Y, Siwila-Sackman E, et al. The HEP Score: A Nomogram-Derived Hematoma Expansion Prediction Scale. Neurocrit Care 2015;23:179-87.

24. Yang M, Du C, Zhang Q, et al. Nomogram Model for Predicting Hematoma Expansion in Spontaneous Intracerebral Hemorrhage: Multicenter Retrospective Study. World Neurosurg 2020;137:e470-8.

25. Ovesen C, Havsteen I, Rosenbaum S, et al. Prediction 
and observation of post-admission hematoma expansion in patients with intracerebral hemorrhage. Front Neurol 2014;5:186.

26. Hall AN, Weaver B, Liotta E, et al. Identifying Modifiable Predictors of Patient Outcomes After Intracerebral Hemorrhage with Machine Learning. Neurocrit Care 2021;34:73-84.

27. Wang X, Arima H, Al-Shahi Salman R, et al. Clinical prediction algorithm (BRAIN) to determine risk of hematoma growth in acute intracerebral hemorrhage. Stroke 2015;46:376-81.

28. Chen S, Zhao B, Wang W, et al. Predictors of hematoma expansion predictors after intracerebral hemorrhage. Oncotarget 2017;8:89348-63.

29. Barras CD, Tress BM, Christensen S, et al. Density and shape as CT predictors of intracerebral hemorrhage growth. Stroke 2009;40:1325-31.

30. Yu Z, Zheng J, Xu Z, et al. Accuracy of Shape Irregularity and Density Heterogeneity on Noncontrast Computed Tomography for Predicting Hematoma Expansion in

Cite this article as: Yu F, Yang Y, He Y, Liu J, Liu H, Liu H. Establishment and evaluation of a nomogram model for predicting hematoma expansion in hypertensive intracerebral hemorrhage based on clinical factors and plain CT scan signs. Ann Palliat Med 2021;10(12):12789-12800. doi: 10.21037/apm-213569
Spontaneous Intracerebral Hemorrhage: A Systematic Review and Meta-Analysis. World Neurosurg 2017;108:347-55.

31. Selariu E, Zia E, Brizzi M, et al. Swirl sign in intracerebral haemorrhage: definition, prevalence, reliability and prognostic value. BMC Neurol 2012;12:109.

32. Ng D, Churilov L, Mitchell P, et al. The CT Swirl Sign Is Associated with Hematoma Expansion in Intracerebral Hemorrhage. AJNR Am J Neuroradiol 2018;39:232-7.

33. Yu Z, Zheng J, He M, et al. Accuracy of swirl sign for predicting hematoma enlargement in intracerebral hemorrhage: a meta-analysis. J Neurol Sci 2019;399:155-60.

34. Li Q, Zhang G, Huang YJ, et al. Blend Sign on Computed Tomography: Novel and Reliable Predictor for Early Hematoma Growth in Patients With Intracerebral Hemorrhage. Stroke 2015;46:2119-23.

35. Zheng J, Yu Z, Xu Z, et al. The Accuracy of the Spot Sign and the Blend Sign for Predicting Hematoma Expansion in Patients with Spontaneous Intracerebral Hemorrhage. Med Sci Monit 2017;23:2250-7. 